\title{
Self-polarization in Storage Rings
}

\author{
E. Gianfelice-Wendt*t \\ Fermilab \\ E-mail: eliana@fnal.gov
}

The possibility of electrons becoming polarized in an uniform field was predicted in the early $60 \mathrm{~s}$ by Loskutov, Korovina, Sokolov and Ternov (Sokolov-Ternov effect of radiative polarization).

The very first experimental observation of beam self-polarization followed in 1968 at ACO in Orsay and therefore this year marks the fiftieth anniversary of that event.

In particular at high energy, $e^{ \pm}$polarization is not really for free and in addition experiments are mainly interested in longitudinal polarization. This is obtained through spin rotators which may lead to depolarization. The $p / e^{ \pm}$high energy collider HERA in Hamburg was the first, and until now the only one, designed for delivering longitudinal $e^{ \pm}$polarization to the experiments.

In this paper, after a historical overview of the theory of radiative polarization in storage rings and its first observations, the unique case of HERAe will be highlighted. Measures needed for fostering high polarization will be illustrated with examples from HERAe operation.

23rd International Spin Physics Symposium - SPIN2018 -

10-14 September, 2018

Ferrara, Italy

\footnotetext{
* Speaker.

${ }^{\dagger}$ This manuscript has been authored by Fermi Research Alliance, LLC under Contract No. DE-AC02-07CH11359 with the U.S. Department of Energy, Office of Science, Office of High Energy Physics.
} 


\section{Sokolov-Ternov effect}

The spin of an electron (or positron) in a homogeneous constant magnetic field has two stationary states, parallel or anti-parallel to the field direction. An electron (or positron) moving on a plane perpendicular to such a field describes circular orbits and emits synchrotron radiation. A part of the emitted radiation is accompanied by a flip of the particle spin direction from parallel to anti-parallel to the field and the other way round. The two processes having slightly different probability, there is an exponential build-up of polarization with rate

$$
\tau_{\mathrm{ST}}^{-1}=\frac{5 \sqrt{3}}{8} \frac{r_{e} \hbar}{m_{0}} \frac{\gamma^{5}}{|\rho|^{3}}
$$

and asymptotic value

$$
\left|P_{\mathrm{ST}}\right|=\frac{\left|n^{+}-n^{-}\right|}{n^{+}+n^{-}}=\frac{8}{5 \sqrt{3}}=92.4 \%
$$

where $n^{+}$and $n^{-}$is the number of electrons (positrons) with spin parallel and anti-parallel to the field respectively. The net polarization direction is anti-parallel to the field for electrons and parallel for positrons.

The very first published paper predicting the effect described above is by Ternov, Loskutov and Korovina [1] which appeared in russian in 1961 and in english one year later. However, it is in the 1963 paper (english translation in 1964), by Sokolov and Ternov [2] that the computation of asymptotic polarization and time constant were carried out by solving the exact Dirac equation.

Sokolov-Ternov formulas were generalized in 1970 by Baier, Katkov and Strakhovenko [3] to the case where spin and motion direction are not everywhere perpendicular, which leads to a reduction of the polarization rate and its asymptotic value.

The conditions described in these papers are somehow realized in particles storage rings. The possibility of polarizing $e^{ \pm}$beams for free was clearly appealing. Actually a $e^{ \pm}$beam in an ideally planar ring accelerator has finite radial and longitudinal dimensions. In addition the machine magnets may have mis-alignments which lead to a finite vertical size too. The effect of the fields seen by the particles in quadrupole and sextupole magnets, which are necessary to focus the beam and correct the natural chromaticity, must be therefore taken into account. The kinematics of the expectation value of the spin operator, $\vec{S}$, is described by the Thomas-BMT equation [4][5]

$$
\frac{d \vec{S}}{d t}=\vec{\Omega} \times \vec{S}
$$

$\vec{\Omega}$ depends in general upon machine azimuth and phase space position, $\vec{u}$. In the laboratory frame and MKS units it is given by

$$
\vec{\Omega}(\vec{u} ; s)=-\frac{e}{m_{0}}\left[\left(a+\frac{1}{\gamma}\right) \vec{B}-\frac{a \gamma}{\gamma+1} \vec{\beta} \cdot \vec{B} \vec{\beta}-\left(a+\frac{1}{\gamma+1}\right) \vec{\beta} \times \frac{\vec{E}}{c}\right]
$$

with $\vec{\beta} \equiv \vec{v} / c$ and $a=(g-2) / 2=0.0011597$ (for $e^{ \pm}$).

By applying Thomas-BMT equation to the spin of particles circulating in a storage ring, Baier and Orlov[6] first pointed out the existence of spin-orbit depolarizing resonances

$$
v_{\text {spin }}=m \pm m_{x} Q_{x} \pm m_{y} Q_{y} \pm m_{s} Q_{s}
$$


$v_{\text {spin }}$ being the spin tune, namely the number of precession per machine turn a spin performs around the periodic solution, $\hat{n}_{0}$, to the Thomas-BMT equation on the machine closed orbit. Those resonances are activated by the stochastic emissions of photons in presence of spin rotators and/or in a non-perfectly planar ring. The resulting spin diffusion is of course particularly important at high energy.

Finally, by using a semi-classical approximation, Derbenev and Kondratenko [7] gave an analytical expression for the asymptotic polarization and the build-up constant including spin diffusion. Following their results, outside from resonances, the asymptotic polarization is oriented along $\hat{n}_{0}$ and its value is

$$
P_{\mathrm{DK}}=P_{\mathrm{ST}} \frac{\oint d s<\frac{1}{|\rho|^{3}} \hat{b} \cdot\left(\hat{n}-\frac{\partial \hat{n}}{\partial \delta}\right)>}{\oint d s<\frac{1}{|\rho|^{3}}\left[1-\frac{2}{9}(\hat{n} \cdot \hat{v})^{2}+\frac{11}{18}\left(\frac{\partial \hat{n}}{\partial \delta}\right)^{2}\right]>}
$$

with $\hat{b} \equiv \hat{v} \times \dot{\hat{v}} /|\dot{\hat{v}}|$.

The corresponding polarization rate is

$$
\tau_{\mathrm{DK}}^{-1}=P_{\mathrm{ST}} \frac{r_{e} \gamma^{5} \hbar}{m_{0} C} \oint<\frac{1}{|\rho|^{3}}\left[1-\frac{2}{9}(\hat{n} \cdot \hat{v})^{2}+\frac{11}{18}\left(\frac{\partial \hat{n}}{\partial \delta}\right)^{2}\right]>
$$

The average is meant over the $6 \mathrm{D}$ phase space. The quantity $\partial \hat{n} / \partial \delta$, with $\delta$ relative energy deviation from the nominal machine energy, describes the effect on the particle spin direction of the trajectory perturbation after photon emission.

Following the Derbenev and Kondratenko papers appearance in the western countries, there were some disputes about the meaning of $\hat{n}(\vec{u} ; s)$ and $\partial \hat{n} / \partial \delta$. Many authors have contributed to settle the question (see for instance [8] [9] [10]) and nowadays $\hat{n}(\vec{u} ; s)$ is understood as the solution to the Thomas-BMT equation satisfying the periodicity condition $\hat{n}(\vec{u} ; s+C)=\hat{n}(\vec{u} ; s), C$ being the machine circumference.

\subsection{Polarization computation}

While the correct definition of $\hat{n}(\vec{u} ; s)$ is of course essential for implementing Derbenev-Kondratenko formulas in a computer code able of evaluating polarization in a realistic machine, implementations attempted so far by perturbation expansion of $\hat{n}(\vec{u} ; s)$ [11], Lie algebra [12] and Fourier series [13] have shown all some limitations.

By linearizing orbit and spin motion it is possible to calculate polarization "analytically" in terms of one turn maps. However, this approach, developed by K. Yokoya and A. Chao and implemented in SLIM [14], can detect only linear resonances.

A statistical approach has been used by J. Kewisch in SITROS [15]. The initially fully polarized beam is tracked and stochastic photon emission is simulated by random emission of "big photons" at user selected machine dipoles. Polarization evolves as

$$
P(t)=P_{\infty}\left(1-\mathrm{e}^{-t / \tau_{p}}\right)+P(0) \mathrm{e}^{-t / \tau_{p}}
$$

with

$$
\frac{1}{\tau_{p}} \simeq \frac{1}{\tau_{\mathrm{BKS}}}+\frac{1}{\tau_{\mathrm{d}}} \quad \text { and } \quad P_{\infty} \simeq \frac{\tau_{p}}{\tau_{\mathrm{BKS}}} P_{\mathrm{BKS}}
$$


where $P_{\mathrm{BKS}}$ and $\tau_{\mathrm{BKS}}$ are the Baier, Katkov and Strakhovenko generalizations of Sokolov-Ternov quantities, namely

$$
P_{\mathrm{BKS}}=P_{\mathrm{ST}} \frac{\oint d s \frac{1}{|\rho|^{3}} \hat{b} \cdot \hat{n}_{0}}{\oint d s \frac{1}{|\rho|^{3}}\left[1-\frac{2}{9}\left(\hat{n}_{0} \cdot \hat{v}\right)^{2}\right]}
$$

and

$$
\tau_{\mathrm{BKS}}^{-1}=P_{\mathrm{ST}} \frac{r_{e} \gamma^{5} \hbar}{m_{0} C} \oint<\frac{1}{|\rho|^{3}}\left[1-\frac{2}{9}\left(\hat{n}_{0} \cdot \hat{v}\right)^{2}\right]
$$

SITROS evaluates $\tau_{p}$ from the fit of $P(t)$ and thus $P_{\infty}$ from $\frac{\tau_{p}}{\tau_{\mathrm{BKS}}} P_{\mathrm{BKS}}$.

More recently E. Forest Polymorphic Tracking Code[16] has been used for a modern implementation of this approach [17].

\section{Measurements of radiative polarization}

The very first observation of radiative polarization was in 1968 [18] at ACO (Anneau de Collisions d'Orsay) which was in operation as $e^{+} / e^{-}$collider from 1965 to 1973. Polarization was measured by exploiting the spin dependent cross section of Coulomb scattering. Figure 1 [19] shows a schematic view of the experimental set-up and the measured loss rate which exhibits the expected behavior, namely losses decrease when polarization increases as expected from the Coulomb scattering cross section.

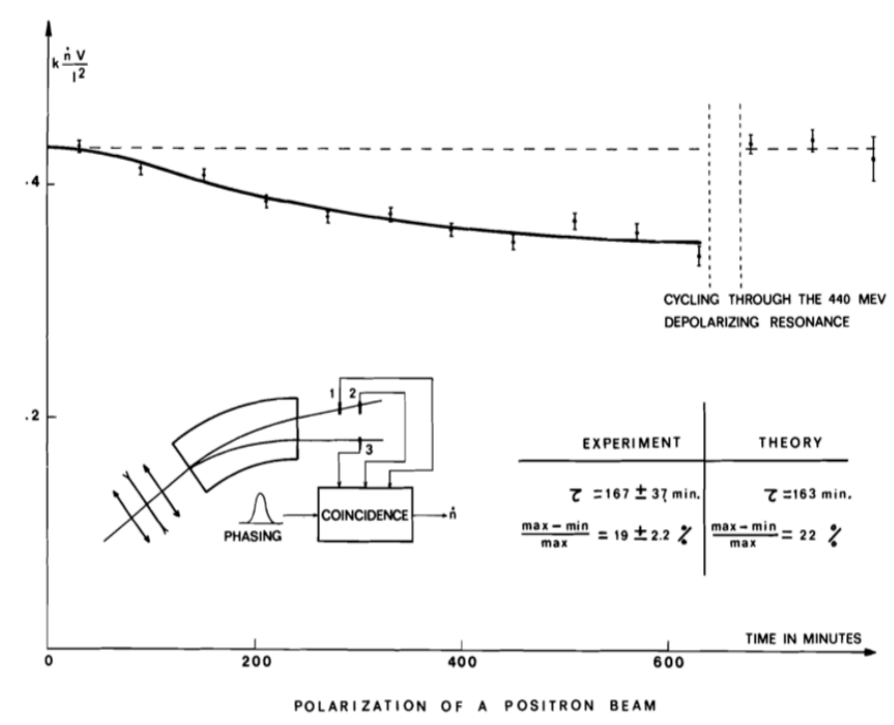

Figure 1: Experimental set-up for the ACO polarization measurement at $536 \mathrm{MeV}$ (from [19]). Positrons exchanging upstreams $7 \%$ energy are separated by the following dipole and intercepted by 2 set of counters. Losses decrease when polarization increases.

Polarization was observed in 1972 also at Novosibirsk storage ring VEPP-2 [20]. Polarization close to $92 \%$ was later measured again at ACO [21] and at VEPP-2M [22] at $500 \mathrm{MeV}$. Figure 2 
(from [23]) shows one polarization build-up measurement at VEPP-2M. The measurement set-up was similar to the one used at Orsay.

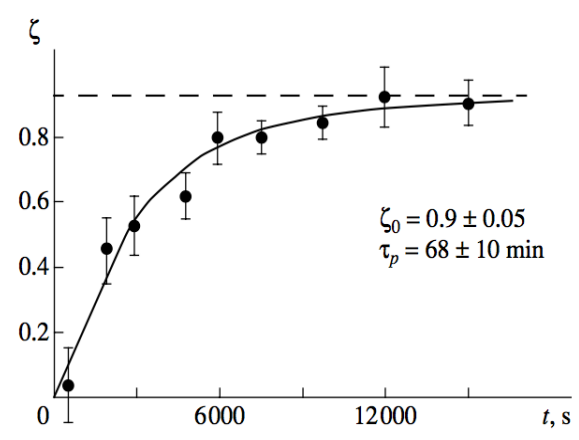

Figure 2: Polarization buildup in VEPP-2M (from [23]).

At VEPP-2M the method of beam energy measurement by resonant depolarization was invented [24]. A time varying longitudinal magnetic field was used for depolarizing the beam. Depolarization occurs when spin precession and field frequency are in resonance

$$
v_{\text {spin }}=\frac{f_{\text {exc }}}{f_{\text {rev }}}+k
$$

Owing to $v_{\text {spin }}=a \gamma$ (in a planar ring, w/o solenoids) the beam energy is given by $E_{\text {beam }}=[k \pm$ $\left.\frac{f_{\text {exc }}}{f_{\text {rev }}}\right] \frac{E_{0}}{a}$.

Large transverse beam polarization was measured later at $3.7 \mathrm{GeV}$ in SPEAR (SLAC) and at $5 \mathrm{GeV}$ in VEPP-4 (Novosibirsk) and DORIS (DESY).

At SLAC the first Compton polarimeter was build for SPEAR2 [25] which allowed more precise and faster polarization measurement wrt the loss rate method. It was based on the spin dependent cross section of Compton scattering of circularly polarized photons from a laser on transversely polarized electrons and was first suggested by Baier and Khoze in 1969.

Figure 3 shows a comparison between measured and computed (by SLIM) polarization for SPEAR [26].

At low energy spin diffusion is small and high polarization could be achieved without having to resort to special corrections. At PETRA (DESY) at $15 \mathrm{GeV}$ a dedicated correction [27] of $\delta \hat{n}_{0}$, the deviation of the periodic solution to the Thomas-BMT equation from the ideal one, was invented and successfully applied to improve polarization from $40 \%$ to $80 \%$. Variations of the same technique, sometime called "harmonic spin matching", were applied later at LEP (CERN), TRISTAN (KEK) and HERAe for improving polarization. Figure 4 [28] summarizes the achieved transverse polarization in various storage rings and shows that spin diffusion becomes stronger at high energies.

At LEP polarization was used solely for precise energy calibrations, but it gave the opportunity for some very interesting observations like the effect of the terrestrial tides on the machine length and hence on the beam energy [29]. 


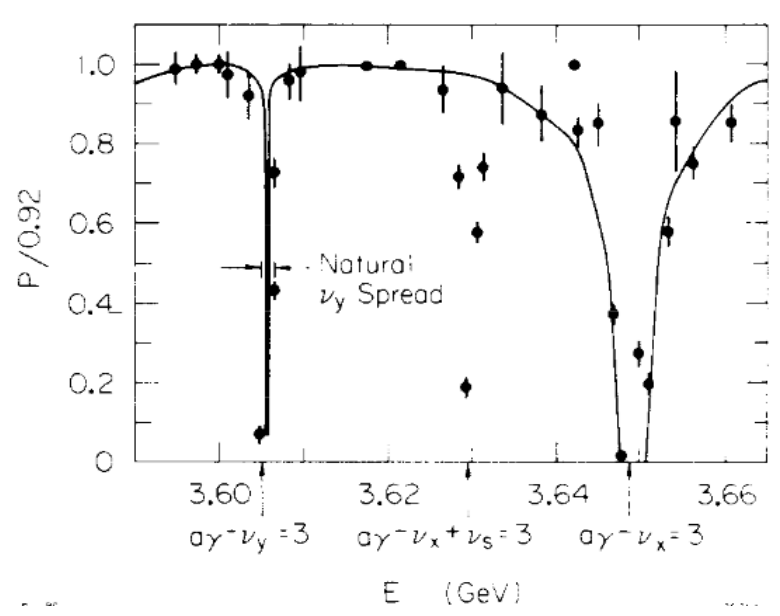

Figure 3: Polarization vs. beam energy at SPEAR (from [26]). Solid line: computation by SLIM; dots: measurements . The second order resonance is missed by the computation with linearized spin motion.

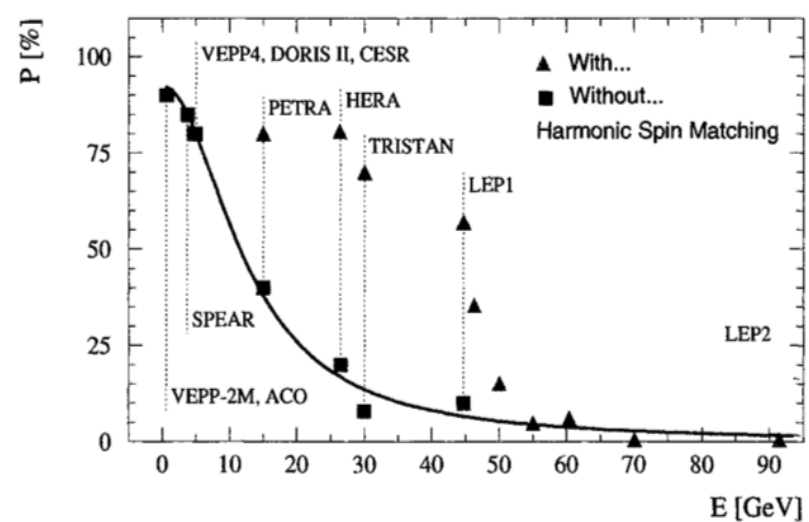

Figure 4: Achieved transverse radiative polarization in various storage rings (from [28]). At high energy $\delta \hat{n}_{0}$ correction is needed to achieve high polarization. At the very large energy achieved at LEP2 the correction was no more sufficient to get high polarization.

\section{Radiative polarization at HERAe}

HERA was a $6.3 \mathrm{~km}$ long $p / e^{ \pm}$collider operating at DESY from 1992 to 2007. Protons were accelerated in a superconducting ring from $40 \mathrm{GeV}$ to $820 \mathrm{GeV}$ and later to $920 \mathrm{GeV}$, while the $e^{ \pm}$ring was designed for accelerating leptons from $12 \mathrm{GeV}$ to $30 \mathrm{GeV}$. In practice, however, the $e^{ \pm}$top energy was limited to about $27 \mathrm{GeV}$. The wish for a $p / e^{ \pm}$collider arose in the early 70s. Ideas based on CERN SPS and PETRA were abandoned in favor of a new facility to be build at DESY [30]. The $e^{ \pm}$ring was conceived from the beginning on for delivering longitudinal beam polarization which is an essential feature of an $p / e^{ \pm}$collider. For this reason spin rotators were integrated in the design and a large number of correctors, beam position monitors (BPMs) and 
independently powered quadrupoles for "spin matching" (see later), was foreseen.

\subsection{HERAe Mini-rotator and spin matching}

For bringing polarization in the longitudinal direction at the Interaction Point (IP), while keeping it vertical in the machine arcs, a pair of $90^{\circ}$ spin rotators is needed before and after the IP. The finally chosen scheme [31] consisted of a sequence of vertical and horizontal bending magnets and fulfilled the requirements of being easily integrable in the machine design, short enough not to require quadrupoles between rotator dipoles and flexible to work between 27 and $35 \mathrm{GeV}$. Spin helicity at the IP was flipped by reversing the sign of the vertical bending angles. The maximum vertical excursion being $\pm 20 \mathrm{~cm}$ (at $27 \mathrm{GeV}$ ), the magnets had to be mounted on remotely controlled jacks for adjusting their elevation. Energy changes larger than about $\pm 100 \mathrm{MeV}$ were possible by manual machine re-alignment.

The presence of rotators breaks the spin transparency of an otherwise planar machine: spin diffusion originates in the quadrupoles between the rotator pair where $\hat{n}_{0} \neq \hat{y}$; vertical dispersion, $D_{y}$, introduced by vertical bending magnets (or by solenoids, through coupling) leads to a finite vertical beam dimension which leads to spin diffusion in the quadrupoles of the whole ring.

Spin transparency for (linear) spin/orbit motion may be recovered by spin matching, which consists in designing the focusing structure so that spin and orbit motion are decoupled [32]. For the HERAe mini-rotator, assuming symmetric focusing around IP and around arc center, this adds 5 optics conditions which in terms of Twiss functions write

$$
\begin{gathered}
\int_{I P}^{s_{R}} d s K \sqrt{\beta_{x}} \cos \mu_{x}=0 \quad \int_{I P}^{s_{R}} d s \hat{n}_{0} \cdot \hat{s} K \sqrt{\beta_{y}} \cos \mu_{y}=0 \\
\int_{A R C S} d s e^{i\left(\psi \pm \mu_{y}\right)} K \sqrt{\beta_{y}}=0 \quad \int_{I P}^{s_{R}} d s D_{x} K=0
\end{gathered}
$$

with $s_{R}=$ rotator entrance and $\psi=v_{s} \times \phi_{b}, \phi_{b}=\phi_{b}(s)$ being the accumulated bending angle. After the HERA luminosity upgrade in 2000-2001, the interaction regions lost their symmetry wrt the IP and the number of spin matching constraints increased to 6 .

\subsection{Effect of misalignments and counter-measures}

In a real machine spin transparency is also randomly broken by magnet misalignments. Usually the most dangerous for beam polarization is the vertical displacement of the quadrupoles which lead to a non-vanishing vertical dispersion and to $\delta \hat{n}_{0} \neq 0$ all along the ring. At HERAe usual closed orbit correction was sufficient to correct also the spurious vertical dispersion. At LEP it was used a "dispersion free" correction aiming to correct closed orbit and spurious dispersion simultaneously.

The correction of $\hat{n}_{0}$ wrt the nominal direction required special care. The expected rms value of the quadrupole vertical displacement was $300 \mu \mathrm{m}$. Simulations showed that after correcting the vertical closed orbit down to about $0.7 \mathrm{~mm}$ it was $\left|\delta \hat{n}_{0}\right|_{r m s} \approx 30 \mathrm{mrad}$ and polarization very low. By introducing extra radial fields through the vertical correctors it is possible to compensate the effect of the displaced quadrupoles. In particular the harmonic expansion of $\delta \hat{n}_{0}$ reveals that the most 
important harmonics of the "spin-orbit" function $f$ [33] are those close to the spin tune

$$
\delta \hat{n}_{0}(s)=-i \frac{L}{2 \pi} \sum_{k} \frac{f_{k}}{k-v_{s}} e^{i 2 \pi k s / C}
$$

and therefore $\delta \hat{n}_{0}(s)$ can be effectively corrected by minimizing those few harmonics. In HERAe up to 8 harmonics were corrected by using 8 closed orbit bumps, each exciting one harmonic at the time while keeping the other 7 unchanged. Figure 5 shows the results of a simulation of the effect of the "harmonic bumps" on HERAe polarization.

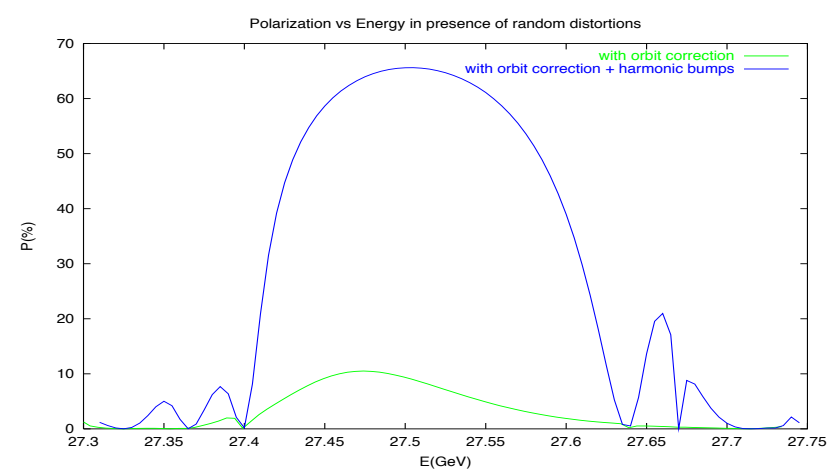

Figure 5: HERAe expected polarization before and after $\delta \hat{n}_{0}$ correction for a typical error realization.
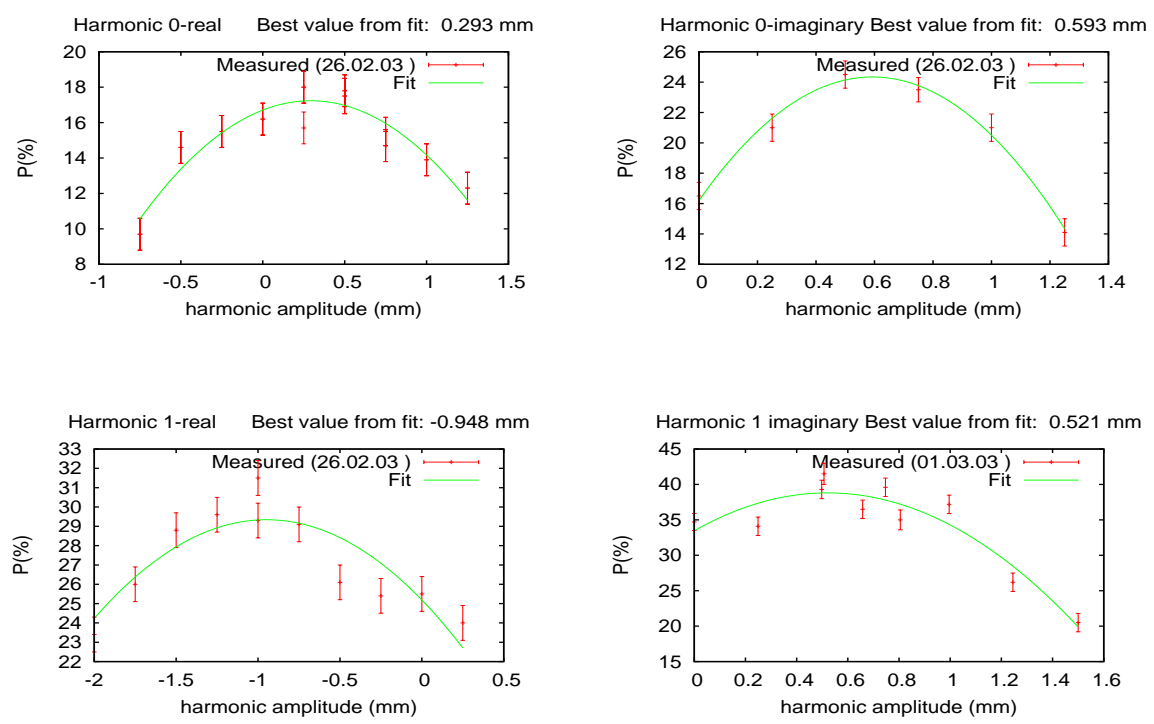

Figure 6: HERAe harmonics optimization in 2003 with 3 pairs of spin rotators.

In HERAe it was not possible to extract the harmonic content of $\delta \hat{n}_{0}$ from the BPMs reading. However, owing to the orthogonality of the components, an empirical optimization, by observing polarization rise/fall, was possible. Figure 6 shows polarization optimization through scanning of the $\delta \hat{n}_{0} 4$ most important harmonics (2003 data). 


\subsection{Beam parameters optimization}

As spin diffusion is larger on the spin-orbit resonances, a key parameter for fostering high polarization is the choice of the working point. In general small fractional parts of the betatron tunes are convenient. HERAe operated with $q_{x} \simeq 0.12$ and $q_{y} \simeq 0.20$. The role of synchrotron tune is more intriguing (see [34], [35], [36], [37]), the spin tune being modulated by the the synchrotron motion.

Another important parameter is the betatron coupling which must be minimized (see for instance [38]). In HERAe there were no skew quadrupoles for a global betatron coupling correction and the observed beam ellipse tilt was corrected through vertical bumps in the arcs sextupoles. Although those bumps alone perturbed $\hat{n}_{0}$ and introduced some spurious vertical dispersion, polarization was improved.

As the spin tune may be not exactly known and resonances are not equally strong, a machine energy scan allows finding the best spot for polarization.

Once high polarization is established a meticulous book keeping may be needed to preserve it, especially if the machine reproducibility is poor: recording of golden orbits; tracking of beam energy changes due to different horizontal corrector settings; run-to-run tracking of harmonic bump settings; analysis of harmonic content of changed vertical corrector kicks.

\subsection{HERAe polarization milestones}

In November 1991 the Compton polarimeter in HERA West was brought into operation and $\simeq 10 \%$ transverse beam polarization was observed at $26.66 \mathrm{GeV}$ w/o dedicated optimizations [39]. In 1992 the collider experiments H1 and ZEUS started data taking; after re-alignment of some machine magnets and correction of the betatron coupling (by vertical bumps through the sextupoles), polarization increased to $\simeq 18 \%$. In June 1992, after dedicated machine tuning (energy and harmonic bumps scans, tunes optimization) it was possible to establish $60 \%$ polarization on routine basis [40]. This success demonstrated the reliability of the polarization simulations and paved the way to the approval of the HERMES experiment to be hosted in the East straight section and to the installation of a pair of Buon-Steffens spin rotators during the 1993/1994 shut down for the experiment.

At the beginning of May 1994 the machine energy was increased to $27.5 \mathrm{GeV}$ for operating the newly installed rotators. Dedicated polarization optimization with rotators still off brought $65 \%$ polarization. However, there was still some nervousness for eventual detrimental effects of the rotators on polarization. On May 4 the rotators were brought into operation and polarization reached 56\% and, after some re-tuning, 65\% [41]. This was the very first time that longitudinal polarization was achieved in a high energy storage ring.

Hermes started data taking in 1995 and, in 1996, the collaboration brought into operation a polarimeter measuring the longitudinal polarization [42]. The longitudinal polarimeter multi-photon mode allowed to measure single bunch polarization with high accuracy in real time providing valuable informations about the effect of the beam-beam interaction on polarization by comparing the polarization of colliding and non-colliding bunches ${ }^{1}$.

\footnotetext{
${ }^{1}$ a small number of non-colliding ("pilot") bunches was kept in each store to correct the luminosity monitor measurement for the bremsstrahlung of $e^{ \pm}$on the residual gas.
} 
Average beam polarization vs. run number from 1996 to 2000 is shown in Figure 7. Despite the increasing beam-beam forces, it was possible to deliver high longitudinal polarization to HERMES. The bad performance in the first half of 1998 was due to lack of time for systematic optimizations.

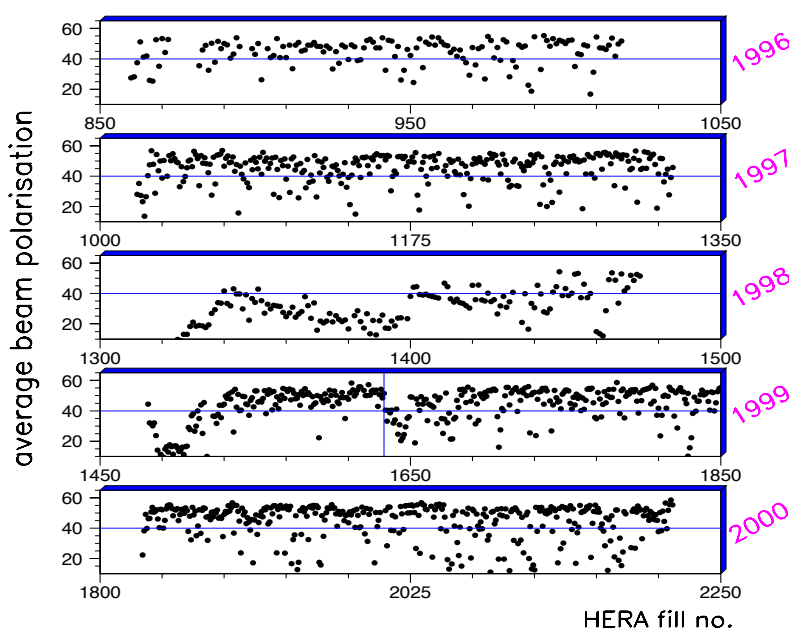

Figure 7: Average longitudinal polarization delivered to HERMES until 2000 (E.C. Aschenauer courtesy).

In 2000/2001 the machine underwent an important luminosity upgrade which involved a rebuilding of the Interaction Regions (IRs). The luminosity upgrade design was of course conceived to maximize the luminosity yield, but wasn't easy on polarization. The compensating solenoids were removed for lack of space. Two more pairs of spin rotators were installed for the collider experiments. The residual $\delta \hat{n}_{0}$ due to the H1 solenoid overlapping with a machine dipole, was corrected with special settings of the $\mathrm{H} 1$ spin rotators. The betatron coupling due to the solenoids was locally corrected by newly installed skew quadrupoles. When in 2001 the machine was brought back into operation, large closed orbit drifts were observed from run to run as well as within the same run. For compensating those drifts, imputable to the weaker magnet supports of the new IR magnets, an orbit feed-back was needed, which effect on polarization was unclear. In February 2003 all 3 rotators pairs were brought into operation and the $e^{+}$beam parameters optimized, first with experiment solenoids turned off and later with them on. Polarization reached $54 \%$ to be compared with a level of 54-60\% expected from non-linear tracking w/o beam-beam forces. Test luminosity was established and polarization reached $50 \%$ w/o further corrections. Details are found in [43],[44].

The main concern of the luminosity upgrade for polarization was the increased beam-beam force on the $e^{ \pm}$beam. A proton bunch colliding with a $e^{ \pm}$bunch acts as a non-linear lenses introducing a shift and spread of the tunes, the effect being larger when the proton bunch intensity increases. The extra lenses is missing for the pilot bunches which therefore have different betatron tunes. As a consequence the colliding bunches may lay on a spin-orbit resonance while the noncolliding are not and the other way around. In addition there is a difference between the effect on $e^{-}$and on $e^{+}$because the proton lenses is focusing for the former and defocusing for the latter. The focusing beam-beam force pushed the $e^{-}$tunes upwards, while for polarization value close to 
an integer are more convenient. Compensating for this effect on the bunch core would had lead to a poor lifetime of the pilot bunches and of the large amplitude particles. The situation was better with $e^{+}$for which the proton lenses was defocusing. In June 2005 "mirror tunes" (i.e. just below the integer) were tried with $e^{-}$. They indeed improved polarization, but at expenses of luminosity and therefore became never operational[45].

\section{Summary}

We have reviewed the milestones of Radiative Polarization in storage rings from the theoretical predictions (by Korovina, Sokolov, Ternov et al.) and the very first observations at ACO (1968), to the unique experience of the HERA $p / e^{ \pm}$collider where radiative longitudinal $e^{ \pm}$beam polarization was for the first time achieved and delivered to the experiments on a routine basis.

\section{References}

[1] I. M. Ternov, Yu. M. Loskutov and L. I. Korovina,"Possibility of polarizing an electron beam by relativistic radiation in a magnetic field”, J.Exptl.Theoret. Phys., 41, 1294-1295 (1961).

[2] A. A. Sokolov, I. M. Ternov, "On Polarization and spin effects in the theory of synchrotron radiation", Sov.Phys.Dokl., vol. 8, pp. 1203-1205 (1964).

[3] V. N. Baier, V. M. Katkov, V. M. Strakhovenko, "Kinetics of Radiative Polarization", Sov.Phys.JETP., vol. 31, pp. 908-911 (1970).

[4] L. H. Thomas, “The Kinematics of an electron with an axis”, Phil. Mag., vol. 3, pp.1-21 (1927).

[5] V. Bargmann, L. Michel and V. L. Telegdi, "Precession of the polarization of particles moving in a homogeneous electromagnetic field", Phys. Rev. Lett., vol. 2, pp. 435-436 (1959).

[6] V. N. Baier and Yu. F. Orlov, "Quantum depolarization of electrons in a magnetic field", Sov.Phys.Dokl. 10 (1966) 1145, Dokl.Akad.Nauk Ser.Fiz. 165 (1965) 783.

[7] Ya. S. Derbenev and A. M. Kondratenko, "Diffusion of particle spins in storage elements", Sov. Phys. JETP, vol. 35, p.230 (1972).

[8] K. Yokoya, "The action-angle variables of classical spin motion in circular accelerators", DESY 86-057 (1986).

[9] S. R. Mane, "Electron spin polarization in high energy storage rings. I. Derivation of the equilibrium polarization", Phys.Review A, vol. 36, pp.105-119 (1987).

[10] G. H. Hoffstätter, M. Vogt and D. P. Barber, "Higher-order effects in polarized proton dynamics", Phys.Rev. ST Accel. Beams, Vol.2, No 11 (1999).

[11] S. R. Mane, "Electron spin polarization in high energy storage rings. II. Evaluation of the Equilibrium Polarization", Phys.Review A, vol. 36, pp.120 (1987).

[12] Yu. Eidelman and V. Yakimenko, "The spin motion calculation using Lie method in collider nonlinear magnetic field", in proceedings of PAC 1991, San Francisco, USA (1991).

[13] K. Yokoya, "Non-perturbative calculation of equilibrium polarization of stored electron beams", KEK Report 92-6 (1992).

[14] A. W. Chao, "Evaluation of radiative spin polarization in an electron storage ring", NIM, vol. 180, p. 29 (1981). 
[15] J. Kewisch, "Depolarisation der Elektronenspins in Speicherringen durch nichtlineare Spin-Bahn-Kopplung", Ph.D. thesis, Phys.Dept., Hamburg Univ. , Hamburg, Germany, 1985.

[16] E. Forest et al., "Introduction to the Polymorphic Tracking Code", KEK Report 2002-3.

[17] Z. Duan et al., "A Monte-Carlo simulation of the equilibrium beam polarization in ultra-high energy electron (positron) storage rings", NIM A, vol. 793, pp. 81-91 (2015).

[18] R. Belbeoch et al., "New experiments with ACO" (in russian), in Proceedings of USSR National Particle Accelerator Conference, Moscow, USSR (1968).

[19] D. Potaux et al., "Status report on ACO", in Proceedings of 8th. International Conf. on High Energy Accelerators,. CERN, pp. 127-131 (1971).

[20] V. N. Baier, "Radiative polarization of electrons in storage rings", Sov. Phys. Uspekhi, vol. 14, p.695 (1972).

[21] J. Le Duff et al., "Experimental evidence for the gradual building up of the polarization of a positron beam in the Orsay storage ring", in Proceedings of of 3rd All-Union Conf. Charged Part.Acc., Moscow, USSR (1972).

[22] S. I. Serednyakov et al., "Study of the radiative polarization of beams in the VEPP-2M storage ring", Sov. Phys. JETP, vol. 44, pp.1063-1067 (1976).

[23] Yu. M. Shatunov, "Polarized beams at storage rings", Physics of Particles and Nuclei Letters, 2006, vol. 3, Suppl. 1, pp. S1-S2.

[24] Ya. S. Derbenev et al., "Accurate calibration of the beam energy in a storage ring based on measurement of spin precession frequency of polarized particles", Particle Accelerators, vol. 10, pp.177-180 (1980).

[25] D. B. Gustavson et al., “A backscattered laser polarimeter for $e^{+} e^{-}$storage rings”. NIM vol.165, pp.177-186 (1979).

[26] A. W. Chao, "Polarization of a stored electron beam", AIP Conf.Proc. 87 395-449 (1982).

[27] R. Rossmanith and R. Schmidt, "Compensation of depolarizing effects in electron positron storage rings”, NIM A, vol. 236, p.231 (1985).

[28] R. Assmann et al., "Spin dynamics in LEP”, Proceedings of 14th Int.Spin Physics Symposium, Osaka, pp.169-178 (2000).

[29] R. Arnaudon et al., "Effects of the terrestrial tides on the LEP beam energy", NIM A, vol. 357, pp.249-252 (1995).

[30] $e / p$ ECFA Working Group, "Study on the proton-electron storage ring project HERA", DESY HERA 80/01 (1980).

[31] J. Buon and K. Steffen, "Hera variable-energy 'mini' spin rotator and head-on ep collision scheme with choice of electron helicity", NIM A, vol. 245, pp.248-261 (1986).

[32] A. W. Chao and K. Yokoya, "An alternative longitudinal polarization scheme for TRISTAN", KEK 81-7 (July 1981).

[33] D. P. Barber et al., "A general spin matching formalism for the suppression of depolarization caused by closed orbit distortion in electron storage rings", DESY 85-044 (1985).

[34] Ya. S. Derbenev et al., "Radiative polarization at ultrahigh-energies”,Part. Acc., vol. 9, pp,247-266 (1979). 
[35] K. Yokoya, "Effects of radiative diffusion on the spin flip in electron storage rings", Part. Accel., vol. 14, p.39 (1983).

[36] C. Biscari et al., "Depolarizing effects of quantum fluctuations and the action of non-linear wigglers on equilibrium polarization level”, Nuovo Cim. B, vol. 81, pp.28-142 (1984).

[37] S. R. Mane, "Synchrotron sideband spin resonances in high-energy electron storage rings", NIM A, vol. 292, pp.52-74 (1990).

[38] E. Gianfelice-Wendt, "Polarization Studies for the eRHIC electron Storage Ring", these Proceedings.

[39] D. P. Barber et al., "The HERA polarimeter and the first observation of electron spin polarization at HERA”, NIM A, vol. 329, pp.79-111 (1993).

[40] D. P. Barber et al., "High spin polarization at the HERA Electron Storage Ring", NIM A, vol. 338, pp.166-184 (1994).

[41] D. P. Barber et al., "The first achievement of longitudinal spin polarization in a high-energy electron storage ring”, Phys.Lett. B, vol. 343, pp. 436-443 (1995).

[42] M. Beckmann et al., "The Longitudinal polarimeter at HERA”, Beam polarimetry at HERA", NIM A, vol. 479 pp.334-348 (2002).

[43] E. Gianfelice-Wendt et al., "First polarisation studies at Hera with 3 spin rotators", DESY-HERA-03-18 (2003).

[44] E. Gianfelice-Wendt et al., "Longitudinal Positron Polarisation in HERA-II", Proceedings of EPAC04, Lucerne, Switzerland (2004).

[45] D. P. Barber et al., "Spin polarisation at DESY”, in ICFA Beam Dynamics Newsletter No.37, pp.88-93 (August 2005). 